\title{
Analysis of retinal sensitivity between acute and recurrent central serous chorioretinopathy assessed by microperimetry
}

Hai-dong Li

Wenzhou Medical University Eye Hospital https://orcid.org/0000-0003-2952-3553

Lifeng Chen

Wenzhou Medical University Eye Hospital

Meng'ai Wu

Wenzhou Medical University Eye Hospital

Bin Zheng ( $\nabla$ goodscing@163.com )

Wenzhou Medical University Eye Hospital

\section{Research Article}

Keywords: central serous chorioretinopathy, retinal sensitivity, microperimetry

Posted Date: September 17th, 2021

DOI: https://doi.org/10.21203/rs.3.rs-903849/v1

License: (a) (i) This work is licensed under a Creative Commons Attribution 4.0 International License. Read Full License 


\section{Abstract}

Purpose To evaluate retinal sensitivity of macular region between acute and recurrent central serous chorioretinopathy (CSC) using microperimetry. Methods A retrospective observational study. Twenty-five eyes of 25 subjects who were diagnosed as either acute or recurrent CSC without any previous treatment were included in this study. All subjects underwent complete ophthalmological examinations including the central retinal thickness (CRT) using spectral domain OCT and the retinal sensitivity assessments of macular region by microperimeter MP-3. The mean global macular sensitivity (GMS) from 64 loci in the $20^{\circ}$ central macular area and local macular sensitivity (LMS) from test locations in affected region of SRD were analysed. Results Twelve eyes of 12 subjects with acute CSC (Group A) and 13 eyes of 13 subjects with recurrent CSC (Group R) were enrolled. The clinical parameters, including age, duration, mean LogMAR best-corrected visual acuity and CRT, were not statistically significant $(p>0.05)$ between Group A and Group R. There were significant differences in GMS and LMS between the two groups. Compared with group A $(24.9 \pm 1.6 \mathrm{~dB})$, the mean GMS of group $R$ was significantly $(p<0.05)$ lower $(23.0 \pm 2.0 \mathrm{~dB})$. In addition, the mean LMS of group R $(19.9 \pm 2.3 \mathrm{~dB})$ was also significantly lower $(p<0.05)$ compared with group $A(21.8 \pm 2.1 \mathrm{~dB})$. Conclusion Eyes with recurrent CSC often show worse retinal function in focal affected macular areas than acute disease. Macular microperimetry analysis may deepen our understanding of CSC natural history and influence decision-making in clinical practice.

\section{Background}

Central serous chorioretinopathy (CSC) is a common chorioretinal disease of unresolved etiology that is characterized by localized and limited serous retinal detachments (SRD) often associated with one or more areas of leakage from the choroid through a defect in the retinal pigment epithelium (RPE) on fluorescein angiography (FA) $[1,2]$. CSC used to be classified in acute and chronic forms. Acute CSC ,a self-limiting disease lasting more than 4 or 6 months, manifests as a dome-shaped area of retinal sensory detachment and usually has a self-limiting process in the majority of cases with few recognized visual sequelae, so observation during the first four months is the most widely used strategy $[1,3]$.Chronic $\mathrm{CSC}$, lasting more, can be a sight-threatening disease leading to legal blindness, for the persistence of SRD that may develop progressive photoreceptor damagement and widespread RPE changes, resulting in permanent loss of visual acuity, so timely effective treatment should be given to stop this progression and improve vision [2, 4]. Nevertheless, the classification relying only on temporal criteria seems too simplistic.

A clear concept of the clinical subtypes of CSC and their exact limits is critical for treatment since it determines the appropriate timing for intervention. But the classical forms of acute and chronic CSC, depending on the duration of symptom and on the presence of non-resolving SRD or RPE changes, are somewhat ambiguous. Daruich et al[5] suggested a newer classification: acute CSC as self-resolving SRD within 4 months from the onset; persistent CSC as acute CSC with duration of SRD longer than 4 months after onset of symptoms, often associated with elongated photoreceptor outer segments on SD-OCT; recurrent CSC as episode of acute CSC following a previous episode with complete SRD resolution; 
Chronic CSC as chronic chorioretinopathy with widespread RPE decompensation with or without SRD, associated or not with active leakage sites; Inactive CSC as patients with history of acute CSC but without SRD at the time of evaluation. The key point of terminology is the distinction between acute and recurrent CSC. As a matter of fact, the duration of symptoms reported from patients is always subjectivity and randomness. For some patients self-describe their disease duration as lasting only a few days, whereas fundus imaging may reveal evidence of prolonged disease, so patient-reported disease duration may therefore be considered unreliable[6]. Beyond the subjective visual acuity examination, retinal sensitivity in macular region can be assessed through microperimetry. Microperimetry, also called 'Fundus-controlled perimetry', is an essential method of examination to assess retinal sensitivity while directly observing the fundus in macular disorders[7-9].The information of microperimetry can help physicians to provide an early treatment if necessary, reducing the chance of permanent vision impairment and CSC chronicity[9]. The purpose of this study was to investigate the difference of macular retinal sensitivity between acute and recurrent CSC using microperimetry, and further to help to select the appropriate management strategy for CSC.

\section{Methods}

This was a retrospective observational case series study conducted at Eye Hospital of Wenzhou Medical University at Hangzhou in China. The procedures used in this research adhered to the tenets of the Declaration of Helsinki, and Institutional Review Board approval for this study was obtained from the local Ethics Committee. Informed consent was obtained from all subjects and all data were anonymized.

Acute CSC was defined as self-resolving subretinal detachment (SRD) within 4months from the first onset of symptoms, and recurrent CSC as an episode of acute CSC following a previous episode with complete SRD resolution[5].All subjects underwent a comprehensive ophthalmic examination including bestcorrected visual acuity (BCVA), slit-lamp biomicroscopic examination, indirect ophthalmoscopy, color fundus photography (CR-2, Canon, Tokyo, Japan), fluorescein and Indocyanine green angiography (HRA Spectralis, Heidelberg Engineering, Heidelberg, Germany), spectral domain OCT(SD-OCT) (OCT Spectralis, Heidelberg Engineering, Heidelberg, Germany) and microperimetry (microperimeter MP-3,NIDEK, Aichi, Japan).

The central retinal thickness (CRT) at the center of the fovea, including the subretinal fluid if present, was evaluated by SD-OCT. Microperimetry assessment was conducted in a dark room with the fellow eye patched and the examination was operated by the same experienced operator. The following testing parameters were used for MP-3囚a 64-stimuli grid overlying the central $20^{\circ}$; Goldmann III stimulus with a duration of $200 \mathrm{~ms} ; 4-2$ full-threshold staircase strategy; $1^{\circ}$ diameter red circle fixation target. The mean retinal sensitivity values for the macula, global macular sensitivity (GMS) from 64 loci in the $20^{\circ}$ central macular area and local macular sensitivity (LMS) from test locations in affected region of SRD, were obtained on the basis of the projected stimuli mean value (Figure 1). 
The results obtained were expressed as mean $\pm S D$ (range). Statistical analyses were performed using Kolmogorov-Smirnov normality test and a t test. $\mathrm{P}<0.05$ was considered statistically significant, and analysis was performed with statistics software (IBM SPSS, version 23; IBM Corp., Armonk, NY, USA).

\section{Results}

Twenty-five eyes of 25 subjects who were diagnosed as either acute (Group A) or recurrent (Group R) CSC without any previous treatment were included in this study. Group A included 3 females and 9 males, with a mean age of $40.3 \pm 9.3$ years (range: $26-55$ ), and the clinical characteristics were shown in Table 1 . Group R included 2 females and 11 males, with a mean age of $41.7 \pm 7.4$ years (range: $28-55$ ), and the clinical characteristics were shown in Table 2. The comparison of clinical parameters between Group A and Group $\mathrm{R}$ were shown in Table 3. The clinical parameters, including age, duration, mean LogMAR BCVA and CRT, were not statistically significant between Group A and Group R. GMS and LMS were both significantly different between the two groups. The mean GMS was significantly $(p=0.018)$ lower for Group R $(23.0 \pm 2.0 \mathrm{~dB})$ compared with Group A $(24.9 \pm 1.6 \mathrm{~dB})$. In addition, the mean LMS was also significantly $(p=0.044)$ lower with Group $\mathrm{R}(19.9 \pm 2.3 \mathrm{~dB})$ compared with Group $\mathrm{A}(21.8 \pm 2.1 \mathrm{~dB})$.

Table 1 Clinical characteristics of cases of acute CSC

\begin{tabular}{|c|c|c|c|c|c|c|c|}
\hline \multirow[t]{2}{*}{ Subject } & \multirow[t]{2}{*}{ Gender } & Age & Duration (d) & BCVA & $\mathrm{CRT}(\mu \mathrm{m})$ & \multicolumn{2}{|c|}{ Macular Sensitivity $(\mathrm{dB})$} \\
\hline & & \multicolumn{2}{|l|}{ (years) } & \multicolumn{2}{|l|}{ (LogMar) } & GMS & LMS \\
\hline 1 & $\mathrm{~F}$ & 50 & 30 & 0.1 & 488 & 25.4 & 22.6 \\
\hline 2 & M & 33 & 7 & 0 & 513 & 24.2 & 22.6 \\
\hline 3 & M & 36 & 14 & 0.1 & 416 & 22.4 & 18.7 \\
\hline 4 & $M$ & 45 & 7 & 0.2 & 344 & 27.2 & 25.6 \\
\hline 5 & $M$ & 31 & 14 & 0.3 & 471 & 23.4 & 20.3 \\
\hline 6 & M & 36 & 4 & 0.2 & 419 & 23.5 & 18.6 \\
\hline 7 & M & 36 & 21 & 0.1 & 522 & 24.2 & 20.1 \\
\hline 8 & $\mathrm{~F}$ & 53 & 30 & 0.1 & 344 & 27.5 & 23.4 \\
\hline 9 & $\mathrm{~F}$ & 55 & 60 & 0 & 459 & 23.6 & 21.4 \\
\hline 10 & M & 36 & 14 & 0 & 302 & 26.6 & 23.5 \\
\hline 11 & M & 26 & 2 & 0 & 398 & 25.5 & 22.2 \\
\hline 12 & $\mathrm{M}$ & 47 & 3 & 0 & 394 & 25.3 & 22.4 \\
\hline
\end{tabular}

Table 2 Clinical characteristics of cases of recurrent CSC 


\begin{tabular}{|c|c|c|c|c|c|c|c|}
\hline \multirow[t]{2}{*}{ Subject } & \multirow[t]{2}{*}{ Gender } & \multirow{2}{*}{$\begin{array}{l}\text { Age } \\
\text { (years) }\end{array}$} & \multirow[t]{2}{*}{$\begin{array}{l}\text { Duration } \\
\text { (d) }\end{array}$} & \multirow[t]{2}{*}{$\begin{array}{l}\text { BCVA } \\
\text { (LogMar) }\end{array}$} & \multirow[t]{2}{*}{$\begin{array}{l}\text { CRT } \\
(\mu \mathrm{m})\end{array}$} & \multicolumn{2}{|c|}{$\begin{array}{l}\text { Macular } \\
\text { Sensitivity(dB) }\end{array}$} \\
\hline & & & & & & GMS & LMS \\
\hline 1 & $M$ & 45 & 10 & 0 & 401 & 25.4 & 22.1 \\
\hline 2 & $M$ & 37 & 30 & 0.1 & 457 & 25.8 & 21.7 \\
\hline 3 & $M$ & 28 & 60 & 0.1 & 480 & 23.2 & 20.8 \\
\hline 4 & $M$ & 36 & 7 & 0 & 514 & 21.9 & 19.7 \\
\hline 5 & $\mathrm{~F}$ & 49 & 30 & 0.2 & 383 & 21.5 & 15 \\
\hline 6 & $M$ & 43 & 30 & 0.1 & 463 & 24.3 & 22.1 \\
\hline 7 & $M$ & 36 & 10 & 0.4 & 434 & 22.7 & 20.1 \\
\hline 8 & $M$ & 45 & 30 & 0 & 447 & 25.2 & 21.8 \\
\hline 9 & $M$ & 55 & 30 & 0.3 & 359 & 19.6 & 17.3 \\
\hline 10 & $M$ & 41 & 60 & 0.3 & 340 & 22.4 & 21 \\
\hline 11 & $M$ & 36 & 21 & 0.1 & 466 & 24.7 & 21.6 \\
\hline 12 & $\mathrm{~F}$ & 51 & 30 & 0.4 & 426 & 22.9 & 19.2 \\
\hline 13 & $M$ & 40 & 7 & 0.1 & 671 & 19.7 & 16.3 \\
\hline
\end{tabular}

Table 3 Comparison of clinical parameters between Group A and Group R

\begin{tabular}{|c|c|c|c|c|c|c|c|}
\hline \multirow[t]{2}{*}{ Group } & \multirow[t]{2}{*}{ Gender } & \multirow{2}{*}{$\begin{array}{l}\text { Age } \\
\text { (years) }\end{array}$} & \multirow[t]{2}{*}{$\begin{array}{l}\text { Duration } \\
\text { (d) }\end{array}$} & \multirow[t]{2}{*}{$\begin{array}{l}\text { BCVA } \\
\text { (LogMar) }\end{array}$} & \multirow[t]{2}{*}{$\begin{array}{l}\text { CRT } \\
(\mu \mathrm{m})\end{array}$} & \multicolumn{2}{|c|}{$\begin{array}{l}\text { Macular } \\
\text { Sensitivity(dB) }\end{array}$} \\
\hline & & & & & & GMS & LMS \\
\hline${ }_{A}^{\text {group }}$ & $3 F / 9 M$ & $40.3 \pm 9.3$ & $17.2 \pm 16.6$ & $0.1 \pm 0.1$ & $423 \pm 70$ & $24.9 \pm 1.6$ & $21.8 \pm 2.1$ \\
\hline group & $2 \mathrm{~F} / 11 \mathrm{M}$ & $41.7 \pm 7.4$ & $27.3 \pm 17.4$ & $0.2 \pm 0.1$ & $449 \pm 82$ & $23.0 \pm 2.0$ & $19.9 \pm 2.3$ \\
\hline $\mathrm{t}$ & - & -0.406 & -1.488 & -1.395 & -0.868 & 2.542 & 2.216 \\
\hline$p$ & - & 0.688 & 0.150 & 0.176 & 0.394 & 0.018 & 0.044 \\
\hline
\end{tabular}

F, female; M, male; BCVA, best-corrected visual acuity; CRT, central retinal thickness; GMS, global macular sensitivity; LMS, local macular sensitivity.

\section{Discussion}


Microperimetry can allow for accurate spatial characterization of visual function providing insightful information about disease severity and progression not reflected by BCVA in a large range of disorders both in clinical practice as well as research[10]. The usefulness of microperimetry in evaluating retinal sensitivity of macular region is well known in CSC. Ojima et al[11] examined macular sensitivity with resolved CSC using Microperimeter-1 (MP-1) and found that eyes of resolved CSC with reduced retinal function at focal affected areas, which were consistent with irregularity of the RPE or with defects of the outer retinal layers. Roisman and colleagues [9] investigated the relationship between retinal sensitivity and persistence of subretinal fluid in acute CSC by MAIA microperimetry and suggested microperimetry with a cutoff of $20 \mathrm{~dB}$ may be a useful test to predict the persistence of subretinal fluid. A study by Parodi et al[12] compared retinal sensitivity obtained with MP1 and MAIA microperimeters in patients affected by retinal dystrophies and in healthy subjects, and the results showed the MAIA microperimeter was more suited to precisely characterizing scotomatous areas with low sensitivity for it had a broader range of retinal light thresholds to be detected than the MP-1. The MP-3 device, which is the successor to the MP-1, is the latest generation of microperimetry and features faster tracking, increased automation and a better structure-function relationship[13]. Interestingly, Balasubramanian et al[8] compared and correlated the retinal sensitivity measurements obtained with MP-3 and MAIA microperimeters among healthy subjects, and as a result, the retinal sensitivity measures higher, but luminance and contrast sensitivity measure lower for MAIA-generated values compared with the MP-3, however, the relationships appeared fairly consistent and application of a standard correction factor allowed the data to be inter-related. To date, there is still a lack of standardization for testing (test-pattern, staircase strategy, adaptation, pupil dilatation), criteria for test repetition as well as reporting standards[10].

In this current study, MP-3 measurement was carried out using 4-2 full-threshold staircase strategy with the standard Goldmann III stimulus size, similar to previous studies[13, 14], and we found that macular sensitivities were significantly lower in recurrent CSC than in acute disease. Although acute episodes are usually self-resolving, $15 \%$ to $50 \%$ of affected eyes may later present recurrence in follow-up varied from 2 to 13 years[5]. The initial BCVA did not differ between recurrent and nonrecurrent cases, but the final BCVA was near-significantly worse in recurrent than nonrecurrent cases[15]. It is plausible that the number of recurrent episodes correlates with visual outcomes in CSC, for repeated episodes and prolonged serous detachment lead to irreversible photoreceptor/RPE damage, and vision loss[2, 16]. Male sex, age, and sleep disorders are risk factors for recurrent or persistent CSC in the natural history[17]. The subfoveal choroidal thickness, nonintense fluorescein leakage at baseline, and history of shift work are independent predictors of CSC recurrence[15]. Microperimetry may be a useful test to predict the persistence of subretinal fluid, allowing the ophthalmologist to use treatment tools earlier, preventing extracellular damage and visual impairment[9]. Although acute CSC usually resolves spontaneously without no long-term subjective symptoms in most cases, some visual sequelae may persist after the resolution of SRD, such as decreased retinal sensitivity[18]. With the automatic tracking system for the retina, the MP-3 microperimeter can project target lights to the retina directly, so even when there is an eye movement, they still stimulate an intended identical retinal location. Recently, Fujita et al[14] demonstrated retinal sensitivity measured using the MP-3 microperimeter was significantly correlated to 
the integrity of ellipsoid zone in resolved CSC. The information of microperimetry may contribute to identify CSC patients at higher risk of recurrence, who could benefit from the most suitable treatment at optimal timing for intervention.

This current study has several limitations. Firstly, the study design was retrospective. Secondly, the sample size was small. Thirdly, the longitudinal follow-up was absent. Finally, there was not the correlation with microperimetry alterations and angiography findings. However, our findings do suggest that recurrent CSC often show worse retinal function in focal affected macular areas than acute disease. This microperimetry information can help doctors to provide an early treatment if necessary to reduce the chance of permanent vision impairment and disease chronicity. Due to the continuous progress in the field of microperimetry, it is believed that the robust tool has great potential for prediction, early detection and treatment-monitoring of macular diseases.

\section{Declarations}

\section{Conflicts of Interest}

The authors declare that they have no conflicts of interest.

\section{References}

1. van Rijssen TJ, van Dijk EHC, Yzer S et al. Central serous chorioretinopathy: Towards an evidencebased treatment guideline. Prog Retin Eye Res. 2019 Nov;73:100770. doi:

10.1016/j.preteyeres.2019.07.003

2. Mrejen S, Balaratnasingam C, Kaden TR et al (2019 Apr) Long-term visual outcomes and causes of vision loss in chronic central serous chorioretinopathy. Ophthalmology 126(4):576-588. doi:10.1016/j.ophtha.2018.12.048

3. Yannuzzi LA (2010 Mar;149(3):361-363) Central serous chorioretinopathy: a personal perspective. Am J Ophthalmol. doi: 10.1016/j.ajo.2009.11.017. PMID: 20172062

4. Breukink MB, Dingemans AJ, den Hollander Al et al. Chronic central serous chorioretinopathy: longterm follow-up and vision-related quality of life. Clin Ophthalmol. 2016 Dec 20;11:39-46. doi: 10.2147/OPTH.S115685

5. Daruich A, Matet A, Dirani A et al (2015 Sep) Central serous chorioretinopathy: Recent findings and new physiopathology hypothesis. Prog Retin Eye Res 48:82-118.

doi:10.1016/j.preteyeres.2015.05.003

6. Ooto S, Hangai M, Sakamoto A et al. High-resolution imaging of resolved central serous chorioretinopathy using adaptive optics scanning laser ophthalmoscopy. Ophthalmology. 2010 Sep;117(9):1800-9, 1809.e1-2. doi: 10.1016/j.ophtha.2010.01.042

7. Bagdonaite-Bejarano L, Hansen RM, Fulton AB (2019) Microperimetry in three inherited retinal disorders. Semin Ophthalmol 34(4):334-339. doi:10.1080/08820538.2019.1622025 
8. Balasubramanian S, Uji A, Lei J et al (2018 Jan) Interdevice comparison of retinal sensitivity assessments in a healthy population: the CenterVue MAIA and the Nidek MP-3 microperimeters. $\mathrm{Br} J$ Ophthalmol 102(1):109-113. doi:10.1136/bjophthalmol-2017-310258

9. Roisman L, Ribeiro JC, Fechine FV et al. Does microperimetry have a prognostic value in central serous chorioretinopathy? Retina. 2014 Apr;34(4):713-8. doi: 10.1097/IAE.0b013e3182a323fe

10. Pfau M, Jolly JK, Wu Z et al. Fundus-controlled perimetry (microperimetry): Application as outcome measure in clinical trials. Prog Retin Eye Res. 2021 May;82:100907. doi:

10.1016/j.preteyeres.2020.100907

11. Ojima Y, Tsujikawa A, Hangai M et al (2008 Jul) Retinal sensitivity measured with the micro perimeter 1 after resolution of central serous chorioretinopathy. Am J Ophthalmol 146(1):77-84.

doi:10.1016/j.ajo.2008.02.016

12. Parodi MB, Triolo G, Morales $M$ et al. Mp1 and MAIA fundus perimetry in healthy subjects and patients affected by retinal dystrophies. Retina. 2015 Aug;35(8):1662-9. doi:

10.1097/IAE.0000000000000504

13. Matsuura M, Murata $\mathrm{H}$, Fujino $\mathrm{Y}$ et al (2018 Mar) Evaluating the usefulness of $\mathrm{mp}-3$ microperimetry in glaucoma patients. Am J Ophthalmol 187:1-9. doi:10.1016/j.ajo.2017.12.002

14. Fujita A, Aoyama Y, Tsuneyoshi S et al. Association between visual function and the integrity of residual ellipsoid zone in resolved central serous chorioretinopathy. Sci Rep. 2019 Aug 27;9(1):12433. doi: 10.1038/s41598-019-48825-7

15. Matet A, Daruich A, Zola M et al. Risk factors for recurrences of central serous chorioretinopathy. Retina. 2018 Jul;38(7):1403-1414. doi: 10.1097/IAE.0000000000001729

16. Loo RH, Scott IU, Flynn HW Jr et al (2002 Feb) Factors associated with reduced visual acuity during long-term follow-up of patients with idiopathic central serous chorioretinopathy. Retina 22(1):19-24. doi:10.1097/00006982-200202000-00004

17. Yu J, Xu G, Chang Q et al. Risk factors for persistent or recurrent central serous chorioretinopathy. J Ophthalmol. 2019 Aug 14;2019:5970659. doi: 10.1155/2019/5970659

18. Ozdemir H, Karacorlu SA, Senturk F et al (2008 Feb) Assessment of macular function by microperimetry in unilateral resolved central serous chorioretinopathy. Eye (Lond) 22(2):204-208. doi:10.1038/sj.eye.6702563

\section{Figures}



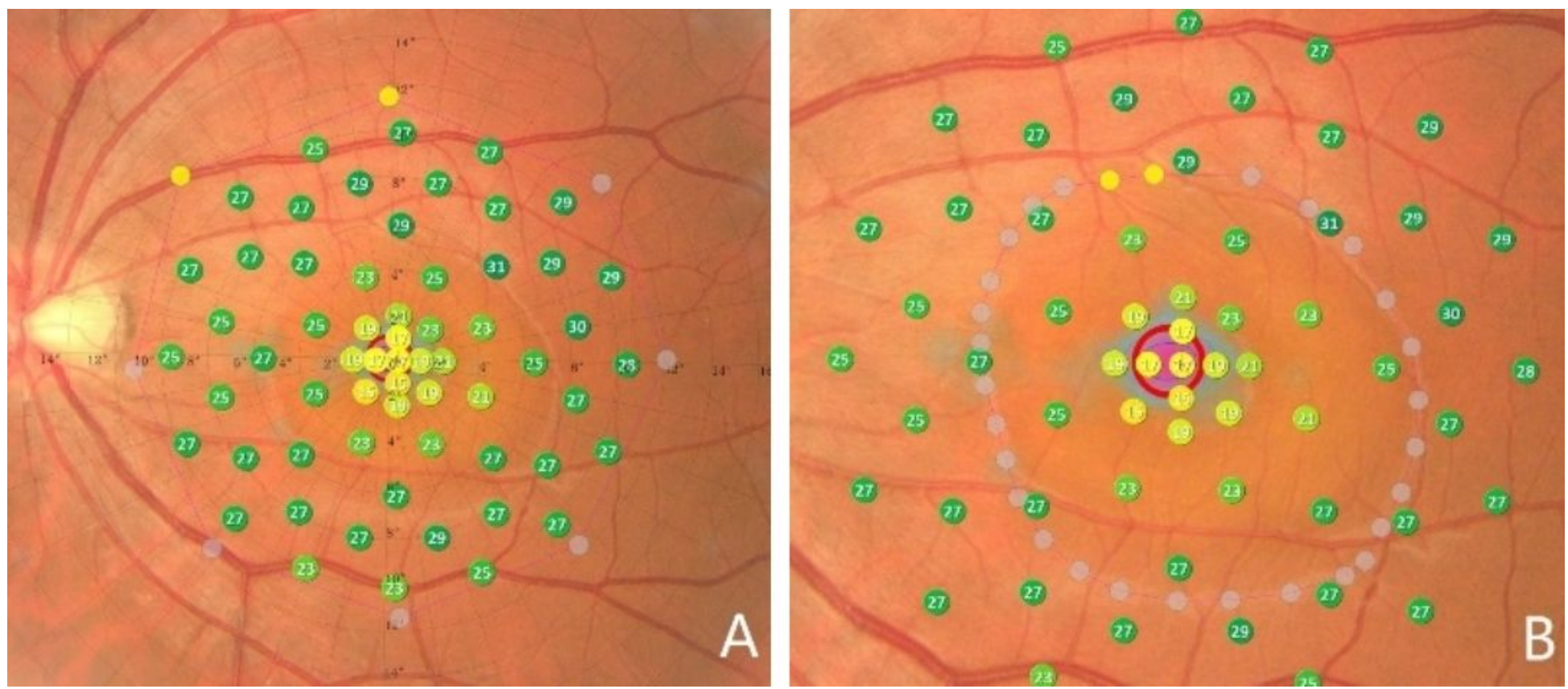

\section{Figure 1}

Microperimetry images of subject 11 in Group R囚the left eye of a 36-year-old male. MP-3 with a grid consisting of 64 stimuli retinal loci in the $20^{\circ}$ central grade $\left(10^{\circ}\right.$ from the foveal fixation $\left.0^{\circ}\right)$. A. GMS in $20^{\circ}$ central grade of macular region. B. LRS in affected region with SRD. MP-3, Microperimetry-3; SRD, subretinal detachment; GMS, global macular sensitivity; LMS, local macular sensitivity. 